\title{
HIDRORESFRIAMENTO NA CONSERVAÇÃO PÓS-COLHEITA DE CEBOLINHA
}

\author{
Amadeu Pimentel Travassos ${ }^{1}$, Eliane Nunes Silva ${ }^{1}$, Renata Ranielly Pedroza Cruz ${ }^{2}$, Christian Raphael
} Delfino Mouzinho Soares ${ }^{2}$, Jean Flaviel de Sousa Macêdo ${ }^{1}$, Wellington Souto Ribeiro ${ }^{3}$

\begin{abstract}
RESUMO - O objetivo deste trabalho foi avaliar o efeito do hidroresfriamento seguido de armazenamento a $12^{\circ} \mathrm{C}$ na manutenção da qualidade pós-colheita de cebolinha. Foram utilizadas para este experimento cebolinhas oriundas de produtor local, colhidas nas primeiras horas da manhã e conduzidas imediatamente ao laboratório, onde foram submetidas à hidroresfriamento. O pré-resfriamento foi efetivo em manter a aparência geral das cebolinhas e os teores de clorofila. Também houve redução na perda de massa fresca e manutenção do balanço hídrico. Portanto, recomenda-se o hidroresfriamento de cebolinha seguido de armazenamento a temperatura de $12^{\circ} \mathrm{C}$.
\end{abstract}

Palavras chave: cadeia de frio, comercialização, pós-produção.

\section{HYDROCOOLING IN POST-HARVEST CONSERVATION OF ONION}

\begin{abstract}
The objective of this work was to evaluate the effect of the hydrocooling followed by storage at $12^{\circ} \mathrm{C}$ in the maintenance of post-harvest quality of chives. Chives from local producers were harvested in the first hours of the morning and immediately taken to the laboratory, were submitted to hydrocooling. Precooling was effective in maintaining the general appearance of chives and chlorophyll content. There was also a reduction in the loss of fresh mass and maintenance of the water balance. Recommended the hydrocooling of chives followed by storage at a temperature of $12^{\circ} \mathrm{C}$.
\end{abstract}

Keywords: cold chain, commercialization, post-production.

\section{INTRODUÇÃO}

A cebolinha (Allium schoenoprasum) é uma hortaliça que tem como partes de interesse econômico caule e folhas, sendo consumido como condimento ou como ingrediente de produtos fitoterápicos, no entanto possui curta vida de prateleira. As folhas da cebolinha são suscetíveis à rápida perda de água após a colheita, acarretando diversas mudanças na aparência, metabolismo e composição da hortaliça, com consequentes alterações na coloração e qualidade nutricional (Wills et al., 2007).

Diante deste contexto, é recomendável o uso de método que retarde os processos de deterioração, o que inclui o pré-resfriamento antes do armazenamento refrigerado. Este procedimento mantém a qualidade de hortaliças, por retirar rapidamente o calor de campo dos produtos antes de serem comercializados, armazenados ou processados, o que aconteceria de forma lenta e gradativa em câmara fria (Becker \& Fricke, 2002). Isto controla mais rapidamente as taxas de respiração, transpiração, a senescência e também a produção de etileno (Kalbasi-Ashtari, 2004).

O hidroresfriamento mostrou-se eficiente no aumento da vida de prateleira de hortaliças folhosas como alface (França et al., 2015) e salva (Alvarez et al., 2007). Em ambos os casos, houve retardamento da perda de massa

\footnotetext{
${ }^{1}$ Graduando em Agroecologia da Universidade Estadual da Paraíba, Campus II, Lagoa Seca, amadeutravassos@gmail.com; lianbela2010@gmail.com; jeanjfsm@gmail.com

${ }^{2}$ Graduando em Agronomia da Universidade Federal da Paraíba, Campus II, Areia, renataranielly426@ gmail.com; agroph@ gmail.com ${ }^{3}$ Dr. em Fitotecnia, UFV. Prof. do bacharel em Agroecologia, curso técnico em Agropecuária e curso técnico em Agroindústria da Universidade Estadual da Paraíba, Campus II - Lagoa Seca, wellingtisouto@ yahoo.com.br
} 
fresca e de água. No entanto, a mesma técnica não foi efetiva para o coentro, havendo degradação de clorofila e amarelecimento de folhas (Oliveira et al., 2015). Em folhas de coentro, a degradação de clorofila e o consequente amarelecimento podem estar relacionados à intolerância desta espécie a baixa temperatura, ocasionada pelo rápido resfriamento. Diferentemente da alface e salsa que são espécies tolerantes. A razão desta intolerância ao resfriamento e a consequente perda de cor das hortaliças folhosas é atribuída ao desvanecimento da cor verde vívida da clorofila $a$ à uma marrom-azeitona, característica da feofitina (Heaton et al., 1996). Este fenômeno é conhecido como feofitinização, onde o magnésio do centro da molécula de clorofila é substituído por hidrogênio. Outro caminho de deterioração é a remoção da cadeia fitol resultando na formação da clorofilida ou feoforbídeo. Às taxas que levam à reação de feofitinização são, geralmente, mais elevadas que outras vias de degradação da clorofila durante o processamento de alimentos. Durante o armazenamento sob congelamento, as baixas temperaturas tendem a aumentar a precipitação de proteínas dos alimentos por provocarem a diminuição do $\mathrm{pH}$, ampliando as taxas de reações de catálises ácidas, como a feofitinização (Martins \& Silva, 2002). Portanto, estas respostas não estão diretamente relacionadas a um gênero ou família. Portanto, vários fenômenos fisiológicos estão envolvidos na tolerância ou intolerância das espécies ao resfriamento - gênero ou espécie, adicionado a outros fatores podem determinar o efeito específico sobre a resposta.

Nos casos de sucesso, o hidroresfriamento promove o aumento da vida útil dos produtos pela rápida redução da temperatura e da desidratação, como consequência da absorção de água durante o processo.

Nesse contexto, este trabalho teve como objetivo avaliar a influência do hidroresfriamento e do armazenamento refrigerado na conservação pós-colheita de cebolinha.

\section{MATERIAL E MÉTODOS}

\section{Matéria prima, localização e determinação do tempo de hidroresfriamento da cebolinha}

O experimento foi conduzido no laboratório de Biologia do Centro de Ciências Agrárias e Ambiental (CCAA) da Universidade Estadual da Paraíba (UEPB). Para este experimento, foram utilizadas plantas de cebolinha oriundas de produtor local colhidas nas primeiras horas da manhã. Após a colheita, as cebolinhas foram conduzidas imediatamente ao laboratório, onde foram arranjadas em maços de 70-80 $\mathrm{g}$ e em seguida medida a temperatura dos maços com termômetro infravermelho. Em seguida, os maços foram imersos em caixas de isopor contendo água com gelo na proporção de $1: 3(\mathrm{v} / \mathrm{v})$ a $4^{\circ} \mathrm{C}$. A cada 5 minutos, foram retirados dois maços e medida a temperatura. O tempo do hidroresfriamento foi determinado com a estabilização da temperatura. Os tratamentos constaram de: controle a seco, controle úmido (submerso em água destilada a temperatura ambiente) e hidroresfriada. Em seguida, as plantas foram armazenadas a $12^{\circ} \mathrm{C}$.

\section{Análise visual e vida de prateleira}

Os maços de cebolinha foram avaliados do momento da coleta e da aplicação dos tratamentos até o fim do período de prateleira, por meio de notas subjetivas de 0 a 3, sendo:

$0=$ nenhum sintoma de murcha, amarelecimento, oxidação ou outro sinal visual indicativo de perda de qualidade,

1 = sintoma intermediário de murcha, amarelecimento, oxidação ou outro sinal visual indicativo de perda de qualidade,

2 = sintomas avançados de murcha, amarelecimento, oxidação ou outro sinal visual indicativo de perda de qualidade e

3 = impróprios à comercialização e decretado o fim da vida de prateleira.

\section{Perda de massa de folhas frescas}

Durante o armazenamento os maços de cebolinha foram pesados diariamente até o término do período de prateleira, estabelecido junto à análise visual. A perda de massa foi estimada pela equação:

$\mathrm{PMF}=[(\mathrm{MFI}-\mathrm{MFF}) \times 100] / \mathrm{MFI}$, em que: PMF = perda de massa de folha $(\%) ;$ MFI = massa fresca inicial $(\mathrm{g})$ e MFF $=$ massa fresca final $(\mathrm{g})$.

Os dados foram transformados em perda de massa fresca acumulada.

\section{Teor relativo de água}

O teor relativo de água das folhas (TRA) foi avaliado antes e a cada 24 horas após os tratamentos. Foi realizado 
o corte de oito seções de aproximadamente $1 \mathrm{~cm}^{2}$ as quais foram pesadas e obtida a massa fresca (F). As mesmas seções foram hidratadas em espuma de poliuretano de dois $\mathrm{cm}$ de espessura até a porcentagem máxima de hidratação (6 horas). As espumas foram mantidas umedecidas com água destilada evitando o ressecamento. Após esse período, as seções foram novamente pesadas e obtidas à massa túrgida $(\mathrm{T})$ e em seguida foram levadas à estufa com circulação forçada de ar à $70^{\circ} \mathrm{C}$ e obtida à massa seca (W).

O teor relativo de água (TRA) das folhas foi estimado conforme descrito por Catsky (1974) com equação citada por Weatherley (1950):

$\mathrm{TRA}=[(\mathrm{F}-\mathrm{W}) /(\mathrm{T}-\mathrm{W})] \mathrm{x} 100$, em que: TRA $=$ teor relativo de água $(\%) ; \mathrm{F}=$ peso de massa fresca (g); $\mathrm{W}=$ peso de massa seca $(\mathrm{g})$ e $\mathrm{T}=$ peso de massa túrgida $(\mathrm{g})$.

\section{Teor de clorofila}

As alterações nos teores de clorofila foram determinadas por espectrofotometria utilizando $1 \mathrm{~g}$ de material macerado em solução extratora de acetona a $80 \%$ e armazenada por $24 \mathrm{~h}$ no escuro a $4^{\circ} \mathrm{C}$, de acordo o método de Lichtenthaler (1987). As leituras foram feitas em comprimento de onda de 646 e $663 \mathrm{~nm}$ e os teores calculados pelas seguintes equações:

$$
\begin{gathered}
\text { Clorofila a }(\mathrm{mg} / 100 \mathrm{~g})=\left[12,21\left(\mathrm{~A}_{663}-2,81 \mathrm{~A}_{646}\right) \mathrm{x}\right. \\
\mathrm{V}] / 1000 \mathrm{x} \mathrm{mg}
\end{gathered}
$$

Clorofila b $(\mathrm{mg} / 100 \mathrm{~g})=\left[20,13\left(\mathrm{~A}_{663}-5,03 \mathrm{~A}_{646}\right) \mathrm{x}\right.$ $\mathrm{V}] / 1000 \times \mathrm{mg}$

Clorofila total $(\mathrm{mg} / 100 \mathrm{~g})=\left[17,30\left(\mathrm{~A}_{663}+7,18 \mathrm{~A}_{646}\right)\right.$ $\mathrm{x} \mathrm{V}] / 1000 \mathrm{x} \mathrm{mg}$

\section{RESULTADOS E DISCUSSÃO}

Segundo Wills et al. (1998), a taxa de resfriamento de um produto depende de vários fatores, dentre eles a taxa de transferência de calor, diferença da temperatura entre o produto e o meio de resfriamento, propriedades termais do produto, forma, tamanho e massa, relação massa"volume. Segundo Brosnan $\&$ Sun (2001), de forma geral, o produto perde calor rapidamente no início do pré-resfriamento seguido por uma lenta taxa de resfriamento. Este mesmo comportamento foi observado no hidroresfriamento de cebolinha (Figura 1). A temperatura da cebolinha antes do tratamento foi de $22,5^{\circ} \mathrm{C}$, reduzindo $73,33 \%$ aos 5 minutos - após esse período houve estabilização da temperatura.

Houve aumento de perda de massa durante o armazenamento da cebolinha a $12^{\circ} \mathrm{C}$, independente da aplicação do tratamento (Figura 2). No entanto, a maior perda de massa foi observada nos maços que compunham o tratamento controle, com $0,27 \%(6,48 \%$ ao dia) de perda de massa por hora. Os maços de cebolinhas pré-resfriados e submersos em água destilada, apresentaram comportamento de perda de massa semelhante, com taxa média de $0,15 \%$ (3,6\% ao dia) de perda de massa por hora.

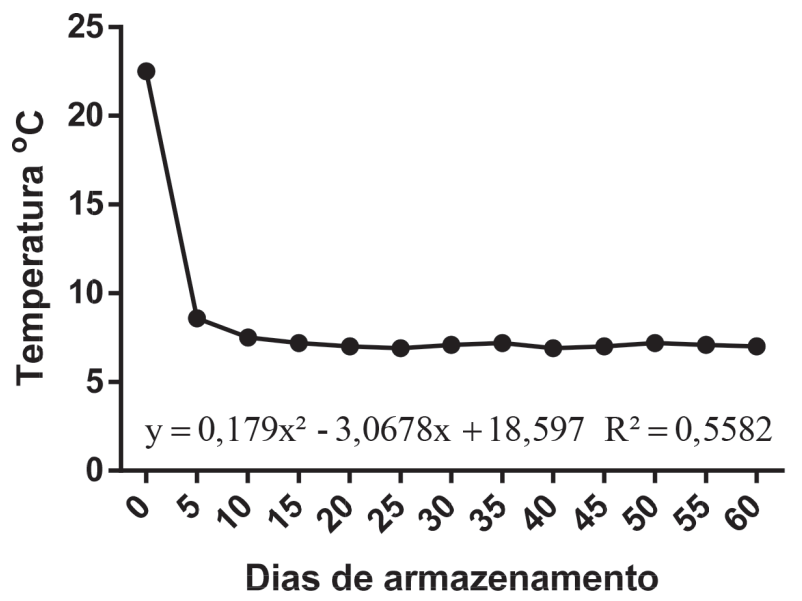

Figura 1 - Valores médios de temperatura de cebolinha em função do tempo de pré-resfriamento.

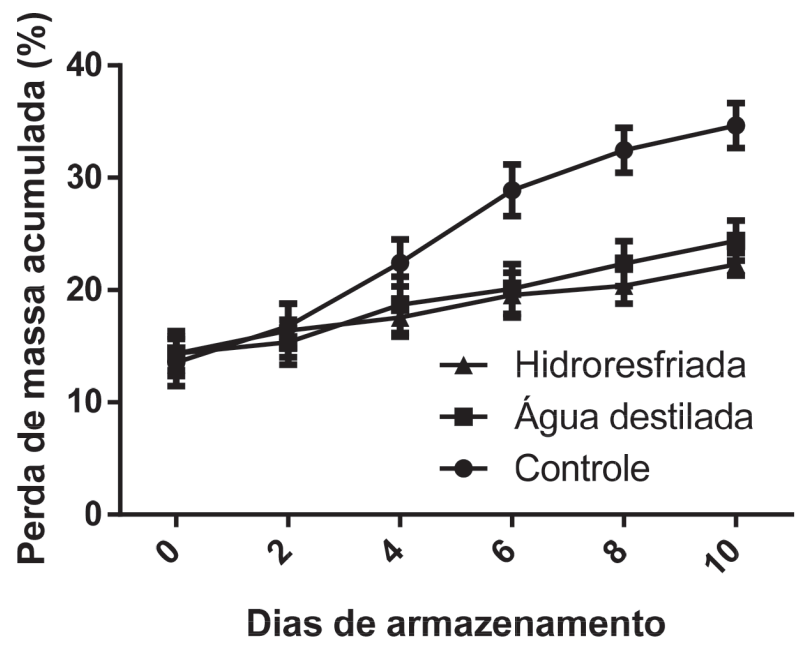

Figura 2 - Perda de massa acumulada de cebolinha durante o armazenamento a $12^{\circ} \mathrm{C}$. 
Estes dados contrastam com os encontrados por França et al. (2015), que observaram maior perda de massa fresca em alfaces hidroresfriados. No entanto, isto pode ser explicado devido à perda de água retida na superfície das folhas e absorvida pelos tecidos durante o hidroresfriamento e não por acelerar o processo de senescência.

Em geral, a perda de massa fresca entre 5\% e 10\% na maioria das hortaliças folhosas acarreta um rápido declínio da qualidade pós-colheita. Porém a porcentagem absoluta de perda de massa depende da exigência do mercado consumidor. $\mathrm{O}$ murchamento e o enrugamento são os principais sintomas da excessiva perda de água, além disso, podem acelerar a deterioração pelo aumento da taxa de algumas reações de origem predominantemente catabólicas (FINGER \& VIEIRA, 1997). Estes sintomas expressaram-se visivelmente no $1^{\circ}, 4^{\circ}$ e $6^{\circ}$ dia durante o período experimental para os tratamentos controle, agua destilada e hidroresfriamento, respectivamente (Tabela 1).

O hidroresfriamento seguido de armazenamento a $12^{\circ} \mathrm{C}$ manteve o balanço hídrico das folhas da cebolinha e superior aos demais tratamentos. As plantas submersas em água destilada e controle a seco apresentaram comportamento semelhante durante $\mathrm{o}$ armazenamento (Figura 3). França et al. (2015) concluíram que o hidroresfriamento da alface durante 5 minutos, foi uma técnica eficaz para manter o equilíbrio hídrico das folhas, promovendo maior tempo de armazenamento.

Segundo Taiz \& Zeiger (2005), este efeito é explicado pela redução do gradiente de pressão de vapor e da taxa de difusão do vapor de água do produto para o ambiente, visto que a perda de umidade é grandemente determinada pela diferença entre a pressão de vapor do produto e a pressão de vapor do ar circundante. Neste experimento, o pré-resfriamento diminui a temperatura do produto, reduzindo a respiração e produção de calor logo após a colheita. Os maços de cebolinha armazenados frios e úmidos tiveram redução no gradiente de pressão em relação ao ambiente diminuindo a perda de água (Figura 1) e consequentemente mantendo o balanço hídrico nas folhas. O que resultou em maços de cebolinha com aparência fresca e aptos a comercialização mesmo cos 10 dias de armazenamento (Tabela 1 e Figura 4).

Não foram observadas alterações visuais na coloração das folhas de cebolinha hidroresfriadas e armazenadas a $12^{\circ} \mathrm{C}$ (Figura 4). Isto pode ser comprovado pela manutenção dos teores de clorofila observados durante $\mathrm{o}$ armazenamento das cebolinhas hidroresfriadas e armazenadas a $12^{\circ} \mathrm{C}$ em comparação àquelas submersas em água destilada e controle a seco (Figura 5).

Na Tabela 1, estão apresentadas as notas subjetivas das cebolinhas pré-resfriadas e controle durante o armazenamento $\mathrm{a} 12^{\circ} \mathrm{C}$. Aos 10 dias de armazenamento, apenas as cebolinhas hidroresfriadas obtiveram nota

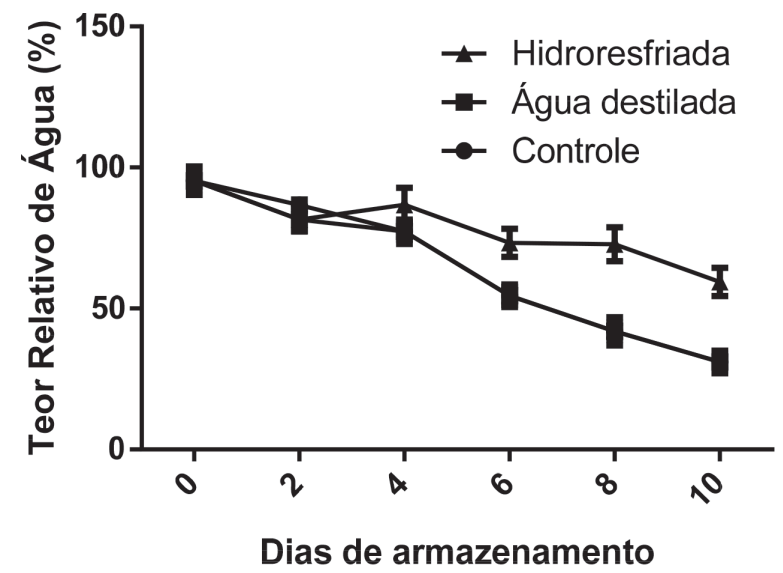

Figura 3 - Teor relativo de água em folhas de cebolinha durante armazenamento a $12^{\circ} \mathrm{C}$.

Tabela 1 - Notas subjetivas das cebolinhas aos 10 dias de armazenamento a $12^{\circ} \mathrm{C}$

\begin{tabular}{cccc}
\hline \multirow{2}{*}{ Dias de armazenamento } & \multicolumn{3}{c}{ Notas subjetivas } \\
\cline { 2 - 4 } & Controle & Água destilada & 0 \\
\hline 0 & 0 & 0 & 0 \\
2 & 1 & 0 & 0 \\
4 & 1 & 1 & 1 \\
6 & 2 & 2 & 1 \\
8 & 3 & 3 & 2 \\
10 & 3 & 2 & \\
\hline
\end{tabular}



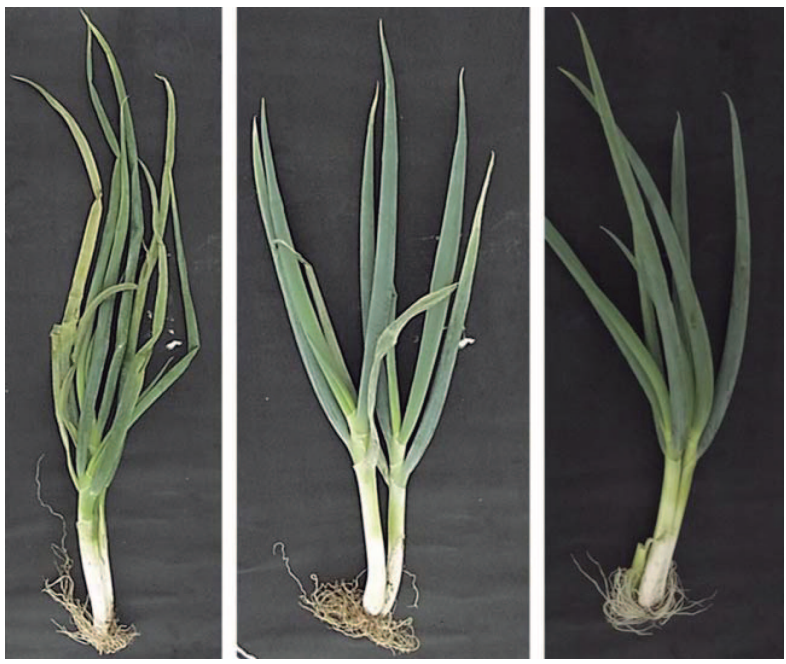

Figura 4 - Aspecto geral das cebolinhas controle (A), submersas em água destilada (B) e hidroresfriadas (C) aos 10 dias de armazenamento a $12^{\circ} \mathrm{C}$.

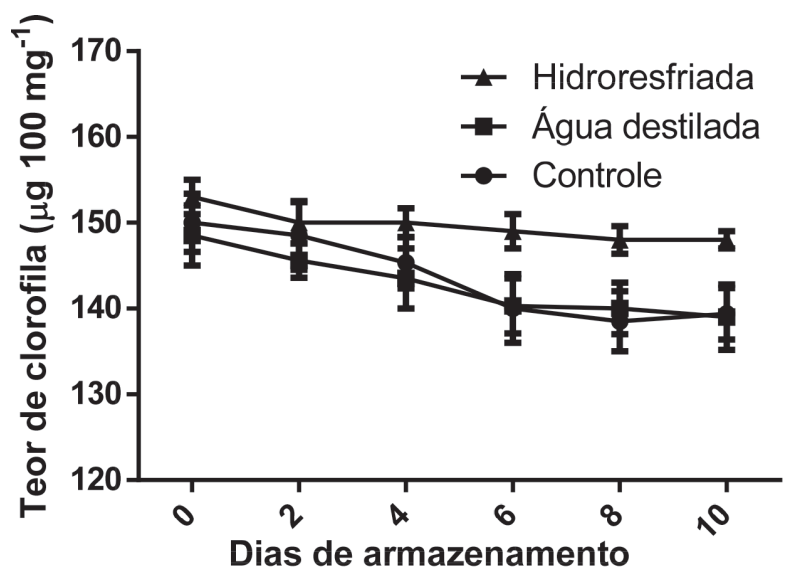

Figura 5 - Teor de clorofila em folhas de cebolinha durante o armazenamento a $12^{\circ} \mathrm{C}$.

2 em sua avaliação, com poucos sinais de senescência e perda de qualidade, caracterizados pela murcha parcial dos maços, amarelecimento e escurecimento"oxidação das extremidades das folhas (Figura 4).

\section{CONCLUSÃO}

Recomenda-se o hidroresfriamento de cebolinha, com tempo ideal de hidroresfriamento de 10 minutos, seguido de armazenamento a $12^{\circ} \mathrm{C}$.

\section{LITERATURA CITADA}

ÁLVARES, V.S.; FINGER, F.L.; SANTOS, R.C.A. et al. Hydrocooling time estimation methods.

International Communications in Heat and Mass Transfer, v.29, n.2, p.165-174, 2002.

BROSNAN, T.; SUN, DA-WEN. Precooling techniques and applications for horticultural products - a review. International Journal of Refrigeration, v.24, p.154-170, 2001.

CATSKY, J. Water content. In: SLAVIK, B. Methods of studying plants water relations. Berlin: Springer - Verlag, 1974, p.121131.

ÁLVARES, V.S.; FINGER, F.L.; SANTOS, R.C.A. et al. Effect of pre-cooling on the postharvest of parsley leaves. Journal of Food,

Agriculture \& Environment, v.5, n.2, p.31-34, 2007.

FRANÇA, C.F.M.; RIBEIRO, W.S.; SILVA, F.C. et al. Hydrocooling on postharvest conservation of butter lettuce. Horticultura Brasileira, v.33, n.3, p.383-387, 2015.

HEATON, J.W.; MARANGONI, A.G. Chlorophyll degradation in processed foods and senescent plant tíssues. Trends in Foods Science \& Technology, v.7, p.8-15, 1996.

KALBASI-ASHTARI, A. Effects of postharvest pre-cooling processes and cyclical heat treatment on the physicochemical properties of "Red Haven Peaches" and "Shahmiveh Pears" during cold storage. Agricultural Engineering International: CIGR Journal, 2004.

LICHTENTHALER, H.K. Chlorophylls and carotenoids: pigments of photosynthetic biomembranes. In: PACKER, L.; DOUCE, R. (Eds.). Methods in enzimology. London: Academic Press, 1987. p.350-381.

MARTINS, R.C.; SILVA, C.L.M. Modelling colour and chlorophyll losses of frozen green beans (Phaseolus vulgaris. L.). International Journal of Refrigeration, v.25, p.966-974, 2002. 
OLIVEIRA, L.S.; DA SILVA, T.P.; FERREIRA, A.P.S. et al. Efeito do hidroresfriamento na conservação pós-colheita de coentro.

Horticultura Brasileira, v.33, n.4, 2015.

TAIZ, L.; ZEIGER, E. Fisiologia vegetal. 3.ed. Trad. SANTARÉM, E.R. et. al. Porto Alegre:

Artmed, 2004. 719p.
WEATHERLEY, P.E. Studies in the water relation of cotton plant. In: The field measurement of water deficits in leaves. New Phytologist, v.49, n.1, p.81-97, 1950.

WILLS, R.B.H.; McGLASSON, W.B.; GRAHAM, $D$. et al. Postharvest: an introduction to the physiology \& handling of fruit, vegetables \& ornamentals. 4.ed. Australia: Cab International, 1998. 262p.

Recebido para publicação em 11/3/2017 e aprovado em 17/5/2017. 